\title{
Overview of an internationally integrated nanotechnology governance
}

\author{
Sheila Devasahayam* \\ Macquarie University, Sydney 2109, NSW, Australia
}

Received: 28 October 2016 / Accepted: 5 January 2017

\begin{abstract}
Nanoscience and nanotechnologies (NTs) have huge potential to improve competitiveness and sustainable development across a wide range of industrial sectors. Public perception of NT related to health, safety and environment (HSE) is critical for the responsible development of NT. It warrants a regulatory regime to demonstrate to the general public whether NTs are safe with regard to HSE. This work explores the feasibility of legal metrology (LM) as a platform to address the impact of NT on legal and socio-economic aspects. It considers whether a LM framework needs to be developed for NT and the associated benefits and risks.
\end{abstract}

Keywords: legal metrology nanotechnology / nano-scale properties / manufactured nanomaterials / nanometrology

\section{Introduction}

Nanoscience and nanotechnologies (NTs) have huge potential to improve competitiveness and sustainable development across a wide range of industrial sectors including in electronics, energy, cosmetics, medical, defence and food \& agriculture sector. The NT market can be segregated into nanomaterials, nanotools and nanodevices.

It has been forecasted that the global NT industry will grow to reach US\$ 75.8 billion by 2020 [1]. Some of Australia's NT applications [2] include coatings, pharmaceuticals, health care, catalysts, advanced ceramics, cosmetics, sunscreens, solar cells, batteries, occupational health and worker safety, environmental safety, medical devices and food (Fig. 1) [3,4].

In this paper, we explore whether a legal metrology (LM) framework for NT is needed in Australia and to recommend if and what form of LM framework is required.

At present, public perception of NT related to health, safety and environment (HSE) is a critical factor that needs addressing. It is anticipated that nanometrology as a tool will enable the industry to meet regulations to protect the workforce and the general public and demonstrate whether NTs are safe with regard to HSE and will provide a platform for the responsible development of NT.

\footnotetext{
$\star$ This journal article is written on the basis of the research results presented during the 2nd Edition Nanotech France 2016 that was held from $1^{\text {st }}$ to $3^{\text {rd }}$ June 2016 in Paris, France.

* Corresponding author: sheila.devasahayam@mq.edu.au
}

Potential gaps [5] in the decentralized and fragmented regulatory regimes across the world have been identified, though considered adequate to regulate the impact of NT to have. Hence there is a general consensus for an internationally integrated NT governance approach to address the ethical, legal and social aspects of the NT [6].

This work explores the feasibility of LM as a platform to address the impact of NT on legal and socio-economic aspects. It considers whether a LM framework needs to be developed for NT and what are the benefits and risks of developing such infrastructure.

The following are the objectives of this work:

- To review the adequacy of current regulatory regimes for NT worldwide.

- To explore a feasibility of an Internationally Integrated NT governance regulatory framework.

- To propose the basis of such framework founded on nanometrology and legal metrology and what form of LM framework is required.

\section{Features of nanotechnology}

NT is one of the four convergent technologies, the other three being the biotechnology and bio-medicine, information technology and cognitive technology, and all may be considered as part of NT [7].

NT can be classified into top-down and bottom-up NTs [8]. The manufactured nanoparticles (MNs) under scrutiny for HSE issues are produced through bottom-up or chemical processes (Figs. 2 and 3). 


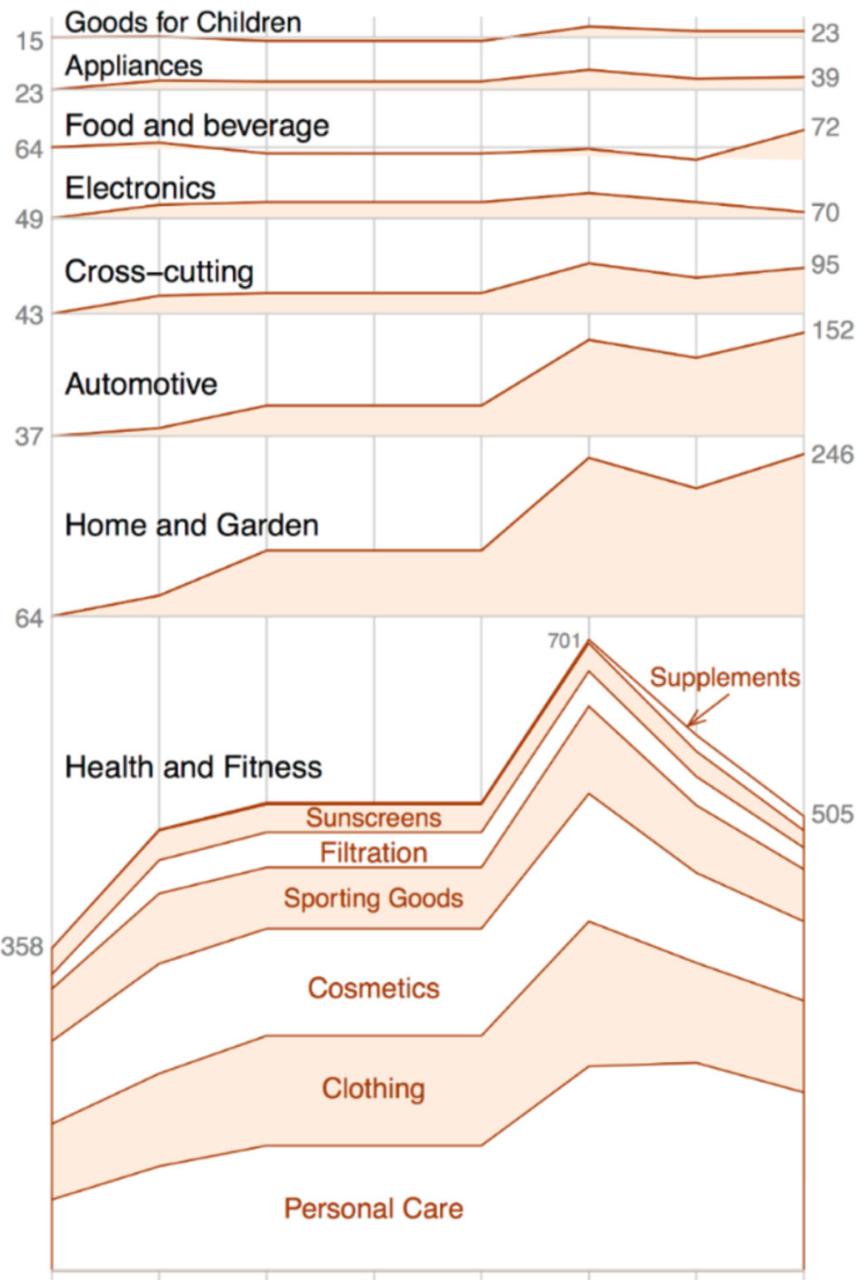

$\begin{array}{llllllll}2007 & 2008 & 2009 & 2010 & 2011 & 2012 & 2013 & 2014\end{array}$

Fig. 1. Nanoparticles in consumer products: number of available products over time (since 2007) in each major category and in the Health and Fitness subcategory [35].

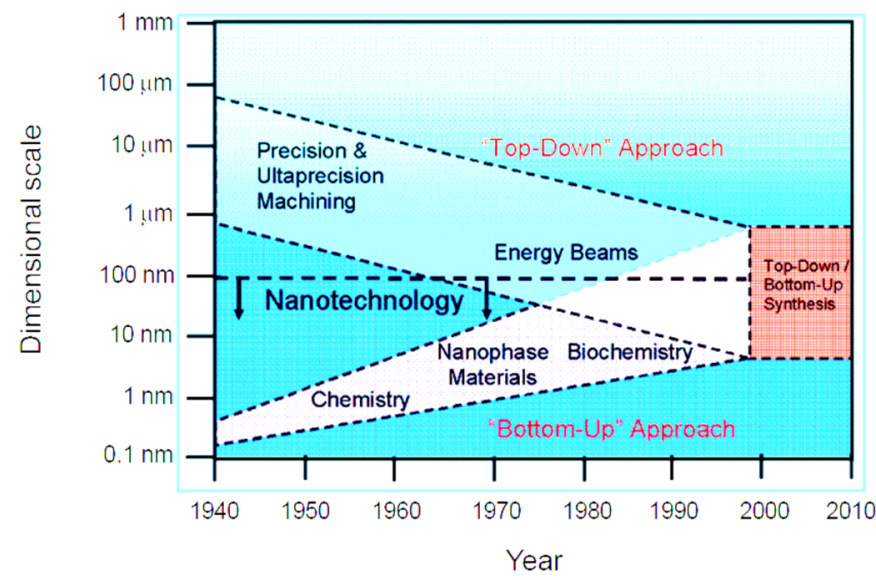

Fig. 2. The use of bottom-up and top-down techniques in manufacturing (Whatmore, 2001) [36].

The specific features of NT are related to: - unique properties of the nano-scale materials; - its interdisciplinary nature.

\subsection{Nano-scale properties}

The main properties of nano-scale materials [9] are as follows: - they have relatively larger surface area when compared to the same mass of material produced in a larger form. This can make materials more chemically reactive and affect their strength or electrical properties

- quantum effects can begin to dominate the behaviour of matter at the nano-scale - particularly at the lower end - affecting the optical, mechanical, electrical and magnetic behaviour of materials.

\subsection{Impacts of nano-scale properties}

The nano-scale properties have given rise to safety concerns as discussed below:

- toxicology studies related to HSE issues of MNs concerns their high surface reactivity due to larger surface area and perhaps the shapes of the particles as well;

- the unique physicochemical properties of the MNs make the risk assessment challenging; and

- the MNs behave very differently from bulk forms of the same material. Their bulk properties cannot be extrapolated to MNs even for the same material in a different geometry, size or environment [10].

Figure 4 shows most common nanoparticles used in consumer products.

\subsection{Intrinsic versus extrinsic nano-scale properties}

Intrinsic properties of MNs concern the material itself. The extrinsic properties are concerned with the functionality associated with the MNs [11]. It is noted, for example, that the intrinsic magnetic properties of the nanomaterial do not vary considerably with size. The new magnetic behaviour different from their bulk is attributed to their extrinsic properties resulting from their interactions and is strongly dependent on their microstructure [12]. Chemical composition leads to intrinsic properties. Size and shape can also underpin some intrinsic properties.

The two distinct nano-scale properties, the high surface area and the unique properties at nano-scale that differ from bulk properties, have been used indiscriminately to describe the impact of nano-materials on HSE.

\subsection{Impact of interdisciplinary nanotechnology}

The interdisciplinary feature of NT poses many challenges due to (a) different terminologies and (b) different measurement approaches and methods used in different branches of different scientific centres and laboratories.

The interdisciplinary aspects of NT have escalated the uncertainties arising from the misunderstanding and difficulty in exchanging technical information. In addition, the unique nano-scale properties, rapid pace of invention, adoption and inadequate science have intensified these uncertainties due to:

- the wide variety of nano-materials and applications;

- limited knowledge and information about their properties and interaction with living and environmental systems; 

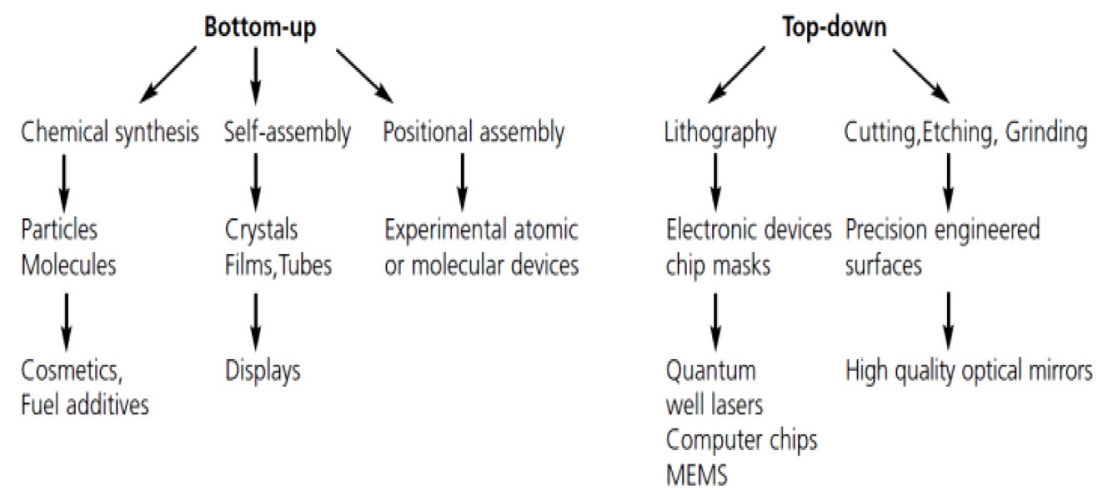

Fig. 3. Convergences of top-down and bottom-up nanotechnologies (Source: [37]).

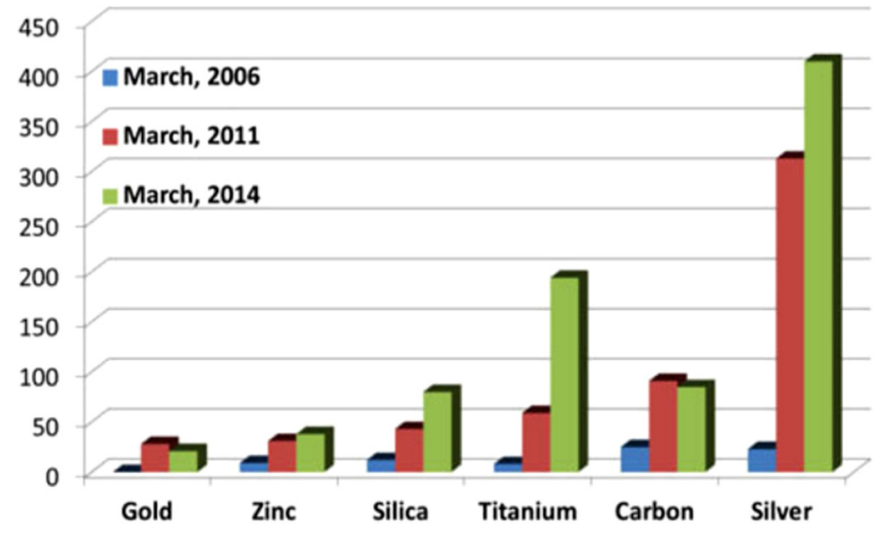

Fig. 4. Nanoparticles used in consumer products. Most common material: silver, carbon (including fullerenes), titanium (including titanium dioxide), silica, zinc (including zinc oxide) and gold [38].

- the proprietary nature of some critical information;

- no consensus in the toxicity of nanoparticles due to the lack of unification of toxicity tests and scarcity of data;

- lack of harmonized standards for guidance (for naming, measuring and identifying nano-materials); and

- potential inadequacy of clear statutory authorities, regulatory frameworks and decentralized regulatory approach to anticipate or respond to any identified risks.

\subsubsection{Harmonized standardization}

The challenges posed by NT have given rise to the need for internationally harmonized standardization in terms of regulatory regimes, nomenclature, measurement protocols and harmonized document standards to provide for responsible development of NT.

ISO TC229 in conjunction with IEC TC113 (TC 113 Nanotechnology standardization for electrical and electronic products and systems) has initiated a line of activities through working groups towards harmonizing standards that focus on the following:

- WG 1 Terminology and nomenclature - Led by Canada;

- WG 2 Measurement and characterization - Led by Japan;

- WG 3 HSE - Led by USA; and

- WG 4 Material Specifications - Led by China.

\subsubsection{Nanotechnology definitions}

The TC229 WG1 had reached consensus on the following definitions of NT (however, they have not been formally approved yet):

Nanotechnology: The application of scientific knowledge to control and utilize matter at the nanoscale, where size-related properties and phenomena can emerge.

Nanoscience: The systematic study and understanding of matter, properties and phenomena related to the nano-scale.

Nano-scale: It is the size range between approximately $1 \mathrm{~nm}$ and $100 \mathrm{~nm}$. A restriction of the definition of nanoscience and NT is that new functionalities are made available by manipulation of matter at this scale or through specificities of the nano dimensions, where the physical, chemical and biological properties of materials differ from those of the bulk matter.

Engineered/manufactured nanoparticle (CEN ISO/TS27687): A particle with three dimensions at the nano-scale, engineered or manufactured by humans on the nano-scale with specific physicochemical composition and structure to exploit properties and functions associated with its dimensions $[13,14]$.

\subsubsection{Nano-scale definition}

Nano-scale definition of $1-100 \mathrm{~nm}$ has incited a series of debates. It is argued that something can be described as nano-scale if it indeed shows a discontinuity, compared with the bulk, with respect to the number of phenomena emerging distinctively different from bulk behaviour. The search for discontinuities and crossovers around the size of a few nanometres revealed that the nano-scale itself is property dependent. The scale also depends on external variables such as temperature and pressure. Scale at which discontinuity compared to bulk emerges for different properties at room temperature is compiled in Table 1. Relative dimensions of different objects are shown in Figure 5: (https://www.weforum.org/agenda/2016/02/ what-is-nanowaste-and-how-will-it-affect-us/).

It is also debated that biological 'unique properties' are not constrained to sizes less than $100 \mathrm{~nm}$. A review of a variety of inorganic nanoparticles greater than $30 \mathrm{~nm}$ 
Table 1. Summary of nano-scale-defining lengths [39].

\begin{tabular}{llr}
\hline Domain & Formula & Typical value nm $^{\text {a }}$ \\
\hline Surfaces & Geometry & 5 \\
Nucleation & Critical nucleus size (6) & 5 \\
Optics and electronics & Bohr radius (7) & 10 \\
Magnetism & Single domain size & 50 \\
Mechanics & Griffith length (13) & 50 \\
Systems & & 100 \\
Electronic & Electron mean free path & 100 \\
Photonic & Evanescent field penetration depth & 10 \\
Fluidic & Debye length (30) & 1000 \\
Mechanical & Phonon mean free path &
\end{tabular}

${ }^{\text {a }}$ At room temperature.

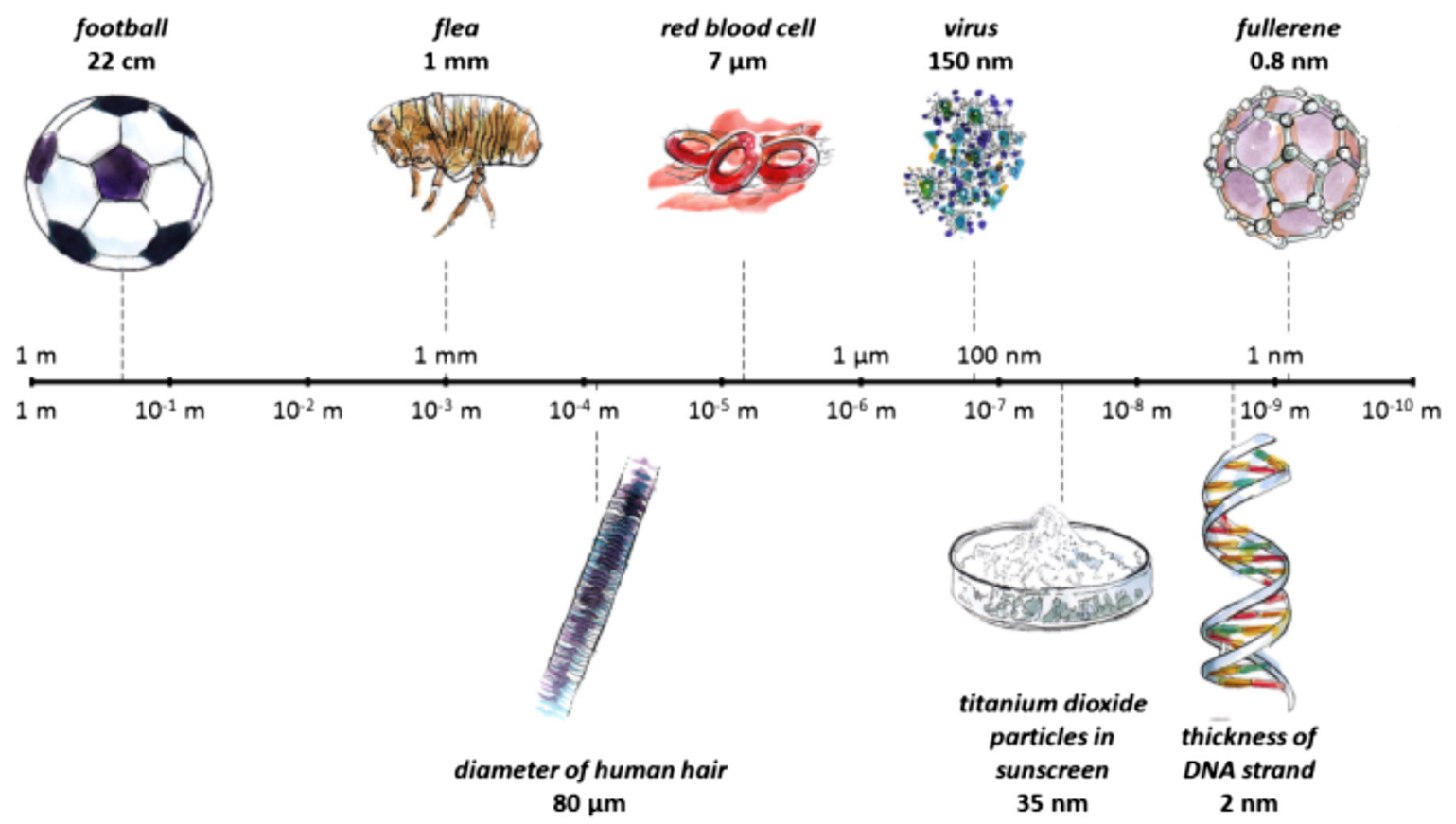

Fig. 5. Dimensions of various objects.

showed that they do not show properties that would require regulatory scrutiny beyond that required for their bulk counterparts [15].

\subsubsection{Prioritizing nanotechnology measurements}

The ISO TC229 and the OECD's WPMN have prioritized the measurement requirements of NT as physical chemical properties of the 'free' engineered MNs during their entire life cycle [16].

Other WPMN projects include:

- OECD database on environmental, health and safety research (Australia);

- research strategies for manufactured nano-materials (Germany);

- safety testing of representative set of manufactured nano-materials (USA);
- manufactured nano-materials and test guidelines (USA);

- cooperation on voluntary schemes and regulatory programmes (Canada);

- co-operation on risk assessment (UK);

- the role of alternative methods in nanotoxicology (UK); and

- exposure measurement and exposure mitigation (USA).

\section{Current regulatory regime's response to health, safety and environmental aspects of nanotechnologies}

It is reported that no country has a comprehensive and co-ordinated oversight system [17] to deal with the impact of NT, including the USA, the EU and Australia. In 
Australia, the current decentralized regulatory regime has prompted the nano-products and nano-devices to be regulated by different regulatory agencies, such as NICNAS, TGA, APVMA and FSANZ.

The US Food and Drug Administration reviewed the immediate health effects of titanium dioxide $\left(\mathrm{TiO}_{2}\right)$ nanoparticles used in sunscreen for consumers, but did not review its impact for aquatic ecosystems when the sunscreen rubs off nor did the US Environmental Protection Agency (US EPA), or any other agencies (Regulation of NT, Wikipedia) [18].

\subsection{Regulatory triggers}

\subsubsection{Classification of manufactured nano-materials}

In Australia, nanoparticles are treated either as a chemical entity or as a product. NICNAS is not required to regulate a nano-entity incorporated into cosmetics if it is already listed as a chemical on the Australian Inventory of Chemical Substance (AICS). The same ingredient in sunscreen will be regulated by the TGA as a product.

The nanoparticles considered as existing chemicals under AICS will not trigger any regulatory oversight over a range of consumer products whether new or existing.

The Toxic Substances Control Act Chemical Substance Inventory (TSCA) of the USA excludes nanomaterials that are not 'chemical substances' ${ }^{1}$ as defined in the law. Size and shape are not normally considered in identifying molecular identity, or in distinguishing one chemical substance from another. It is not clear if a nanoparticle consisting of a core inorganic material coated by an organic material would qualify as a TSCA 'chemical substance', or how nanotubes or fullerenes, with clear chemical identities in terms of chemical composition and crystal structure, but with variable properties due to differences in size or shape will be regulated.

In Europe, whether a nano-material is a new or existing substance $^{2}$, is based on whether it is listed in The European Inventory of Existing Commercial Substances (EINECS). Substances in nano-form which are in EINECS (e.g. $\mathrm{TiO}_{2}$ ) shall be regarded as existing substances. Substances in nano-form which are not in EINECS (e.g. carbon allotropes other than those listed in EINECS) shall be regarded as new substances.

\subsubsection{Regulatory triggers based on weight or volume thresholds}

Regulatory triggers depend upon thresholds determined by weight or volume being met. With the current scientific knowledge, it is not known whether these thresholds are

\footnotetext{
1 TSCA defines a 'chemical substance' as 'any organic or inorganic substance of a particular molecular identity' that is not a mixture (TSCA Sect. 2).

${ }^{2}$ Substance means a chemical element and its compounds in the natural state or obtained by any manufacturing process, including any additive necessary to reserve its stability and any impurity deriving from the process used, but excluding any solvent which may be separated without affecting the stability of the substance or changing its composition.
}

appropriate and meaningful. At present, low production levels of some NMs may not meet the thresholds criterion to trigger regulatory frameworks to be applied.

\subsubsection{Regulatory triggers based on nano-scale definition}

There is a concern that nano-materials just outside the agreed 'nano-scale' definition, that is 1 to $100 \mathrm{~nm}$, will not be regulated as a discrete category, even if they pose the same threat to HSE [19] as their counterparts. There is also regulatory concern related to the distribution of MNs some within and some just outside the nano-scale limits.

\subsubsection{Regulatory responsibilities}

In the USA (EPA-TSCA), it is the responsibility of the regulators to prove that a chemical is harmful before it can be restricted or removed from the market. In Europe (REACH), it is up to the producer to demonstrate that a chemical is safe before it enters the market. In Australia, the TGA has adopted the approach where the burden of proof lies with the manufacturer to show that the therapeutic good is safe.

Some industries have developed (or are developing) their own risk management systems, defining best practises and procedure for safety control and handling of nanomaterials in occupational settings, for example, the DuPont/Environmental Defence NanoRisk Framework and the Certifiable Nanospecific Risk Management and Monitoring System (CENARIOS).

\subsubsection{Regulatory gaps}

Hodge et al. have summarized the potential regulatory gaps world wide as follows:

- distinction between 'New' or 'Existing' Substances or Products;

- regulatory triggers based on weight or volume;

- knowledge of presence or implications of presence of nano-materials;

- adequacy of risk-assessment protocols and conventional techniques;

- research and development exemptions; and

- international documents and documents sourced outside regulators (adequacy of these references).

The OECD recently published a series of five reports on nano-materials and nanowaste that describe possible methods of disposal, hazard evaluation and recycling. There is yet no consensus on the toxicity of nanoparticles due to lack of unification of toxicity tests. The current hypotheses that the nanoparticles more toxic than the fine-sized counterparts are based on systematic evaluation of $\mathrm{TiO}_{2}$, carbon black and diesel particles based on paucity of data [20]. There is an initiative to propose a new subcategory of toxicology, namely nanotoxicology to address gaps in knowledge and to specifically address the special problems likely to be caused by nanoparticles [21]. Surprisingly, there are no policies or frameworks currently in place to tackle this growing issue.

Such gaps are likely to continue alongside the development and commercialization of increasingly complex second and third generation materials based on 
NTs. It is anticipated that many unknown extrinsic as well as the intrinsic properties of MNs may end up stressing the established regulatory frameworks to breaking point.

Different sets of standards arising from independent development of laws and standards by different countries can impede NT development in addition to creating trade barriers. Hence, there is a general consensus for an internationally integrated NT governance approach to address the ethical, legal and social aspects of NT.

\section{Nanometrology}

The measurement requirement of NT has led to the development of nanometrology, a key enabler for the advancement of nanoscience and exploitation of NTs.

According to Whitehouse [22], nanometrology should mean the measurement of physical phenomena by means which employ distance/deflection techniques at the nanoscale of size. The concept of accuracy should relate to the physical [23] phenomena being measured, not just the distance/deflection output.

Australian nanometrology programmes involve establishing physical standards and instruments capable of transferring Australia's realization of the metre using known optical wavelengths of light, down to nano-metre measurements to the NT community via a chain of comparisons. A metrological scanning probe microscope (SPM), to calibrate artefacts such as gratings, grids and step height standards directly in terms of optical wavelengths, is being developed.

These artefacts would then be used to calibrate a wide range of standards and instruments used in the NT community such as SPM, electron microscopes and other reference standards/materials. International traceability across the nations is established through inter-comparisons between the national measurement systems.

For chemical measurements, there is no internationally agreed realization or prototype of the mole and so traceability is usually achieved through reference materials and reference methods. The Consultative Committee for Amount of Substance (CCQM) conducts international comparisons to establish equivalence among measurements made by national metrology institutes.

The Australian nanometrology programme coordinates the physico-chemical safety testing programme [24]. It plays an active role in ISO TC229 through NT-001, the Standards Australia's mirror committee to ISO TC229 [25], and is also active in APEC and VAMAS programmes.

The challenges for nanometrology include:

- to be able to measure any physical or chemical property of structures and devices in three dimensions at the nanometre scale. At present, many measurements are effective in two dimensions (or 2.5 dimensions), and there is often a trade-off between measurement sensitivity and spatial resolution;

- to ensure the results of measurements are expressed in legal units and the errors of the measurements are known with a specified probability and be comparable to the inter-atomic distances;

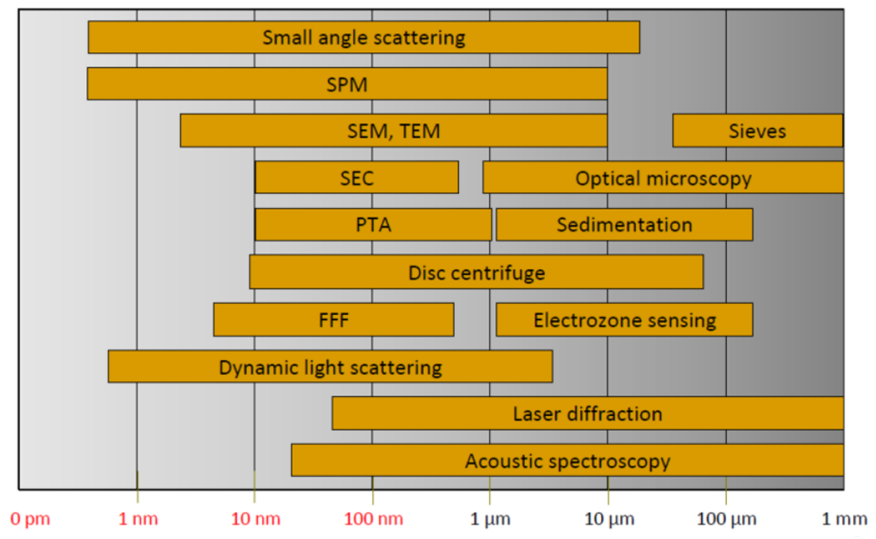

Fig. 6. Size-measuring devices.

- often measuring instruments do not exist to cover the full range of measurement values, and some measuring problems fall between the ranges of available instruments where there is no overlap (Fig. 6, Tab. 2). This is especially true for the transition region between microscale and nano-scale metrology; and

- to collect multiple parameters for example, chemical, spatial and mechanical data simultaneously. This might be achieved by integrating measurement techniques in a multitasking SPM with functionalized tips capable of providing spectroscopic data with nano-metric resolution (Fig. 7).

Within the regulatory context, the nanometrology group aims to enable evidence-based regulatory decisions and reviews, by facilitating accurate and reliable measurement as well as to facilitate harmonized standards.

\section{Australia's national measurement system}

Australia's national measurement system within the global measurement system is coordinated and administered by the National Measurement Institute (NMI) under the National Measurement Act 1960 (Cth) (the Act), and The National Measurement Regulations 1999 (Cth). The NMI also coordinates national activities with a number of commonwealth government agencies under memoranda of understanding (MOU), for example, the Australian Customs Service, FSANZ and DAFF. LM's international harmonization requirements of measurements and regulations are achieved through CIPM MRA and OIML MAA for pattern approval (PA) of measuring instruments and closer integration with metre convention and ILAC to deliver a global measurement system.

\subsection{Measurement traceability}

Measurement traceability (Fig. 8) and confidence in measurements are the key principles of metrology [26], at whatever accuracy and for whatever physical quantity, to ensure that the results of measurements are expressed in legal units with known uncertainties.

The Australian legal measurements are expressed in the International System (SI) of units. 
Table 2. Measurement techniques for nanoparticles [40].

\begin{tabular}{|c|c|c|c|}
\hline Technique & Measures & Sample & Sensitivity \\
\hline $\begin{array}{l}\text { Transmission Electron } \\
\text { Microscopy (TEM) }\end{array}$ & $\begin{array}{l}\text { Particle size and } \\
\text { characterization }\end{array}$ & $\begin{array}{l}<1 \mu \mathrm{g} \text { has to be prepared } \\
\text { as a thin film and be } \\
\text { stable under an electron } \\
\text { beam and a high vacuum }\end{array}$ & Down to $1 \mathrm{~nm}$ \\
\hline
\end{tabular}

Scanning Electron

Microscopy (SEM)

Atomic Force

Microscopy (AFM)

Photon Correlation Spectroscopy (PCS)
Particle size and characterization

Particle size and characterization

Average particle size and size distribution
Sample must be conductive or sputter coated, easier to prepare than TEM sample Samples must adhere to $1 \mathrm{~nm}$ to $8 \mu \mathrm{m}$ a substrate and be rigid and dispersed on the substrate. The appropriate substrate must be chosen. Air or liquid samples

Sample must be a very dilute suspension
Nanoparticle Surface Area Monitor (NSAM)

Condensation Particle Counter (CPC)

Differential Mobility
Analyzer

Scanning Mobility

Particle Sizer (SMPS)

Nanoparticle Tracking

Analysis (NTA)

X-Ray Diffraction

(XRD)

Aerosol Time of Flight

Mass Spectroscopy

Aerosol Particle Mass

Analyzer (APM)
Human lung-deposited Aerosol, concentrations surface area of nanoparticles Number of concentrations of particles

Particle size distribution

Particle size distribution

Particle size and size distribution

Average particle for a bulk sample

Particle size composition

Particle mass 0-10,000 $\mathrm{\mu m}^{2} / \mathrm{cm}^{3}$, temp $10-35{ }^{\circ} \mathrm{C}$

Aerosol, concentrations 0-100,000 particles $/ \mathrm{cm}^{3}$, can be in a flow, higher temps. to $200{ }^{\circ} \mathrm{C}$ possible Aerosol

Aerosol, can be a concentrated sample of $1,000,000$ $2,400,000$ particles $/ \mathrm{cm}^{3}$ $500 \mu \mathrm{l}$ suspension, temp $5-50{ }^{\circ} \mathrm{C}$, wide range of solvents can be used

Larger crystalline samples ( $>1 \mathrm{mg}$ ) required Aerosol

\section{d}

Down to $1 \mathrm{~nm}$

100-3000 nm

Aerosol sample with Equivalent to particle density approx. $1 \mathrm{~g} / \mathrm{cm}^{3}$

3-1000 nm

10-1000 nm

$30-580 \mathrm{~nm}$
Notes

Additions to TEM can provide more information, e.g. Scanning Transmission Electron Microscopy (STEM). High-Resolution TEM (HRTEM) or in-situ measurements as Environmental TEM

Down to $1 \mathrm{~nm}$ Can be used in-situ as Environmental SEM

A form of Scanning Probe Microscopy (SPM). Requires less time and cost than SEM and TEM

$1 \mathrm{~nm}$ to $10 \mu \mathrm{m}$

Based on Dynamic Light Scattering, an extension of the technique is Photon Cross Correlation Spectroscopy (PCCS) for high concentration opaque suspensions giving particle size and stability of nanoparticles

Down to $10 \mathrm{~nm}$ Similar to an Electrical Aerosol Detector (EAD)

2.5 to $>3000 \mathrm{~nm}$ Can be used for a flow, hand held models available

Down to $3 \mathrm{~nm}$ Can be combined with other techniques to create Tandem DMA or DMPS

Uses an electrostatic classifier and a CPC, can also add DMA

Use with DLS or PCS

Can identify individual crystals

The efficiency of this method is less for smaller particles
Gives only mass information and is not dependent on particle size or shape 


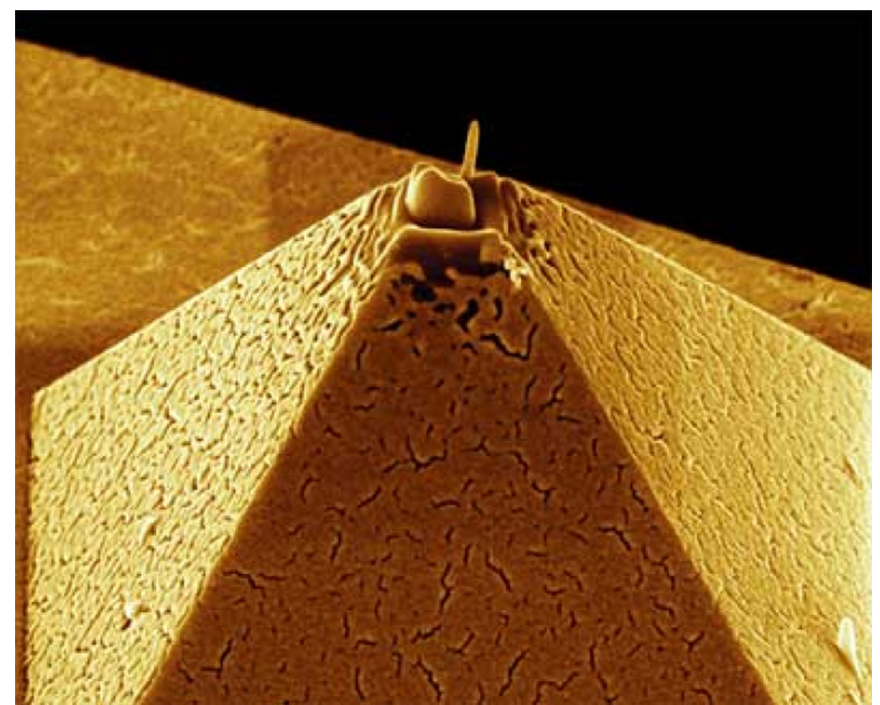

Fig. 7. Tip of an atomic force microscope (AFM) measuring at nano-scale.

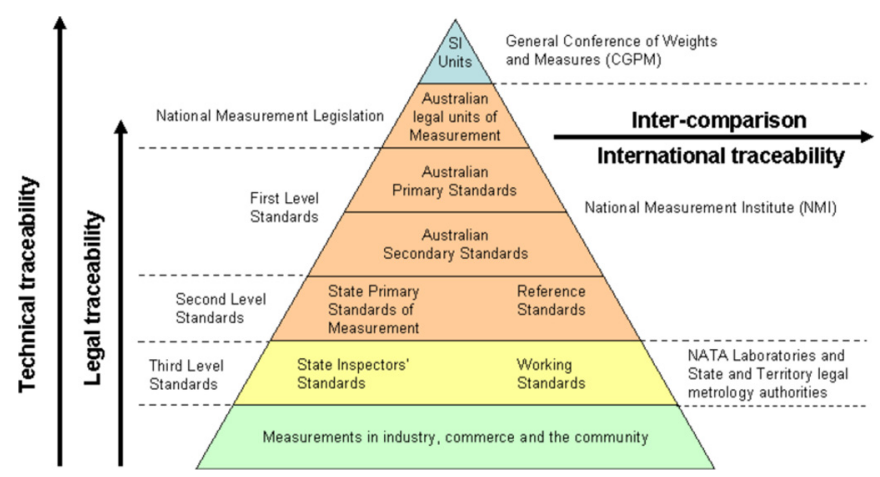

Fig. 8. Measurement traceability.

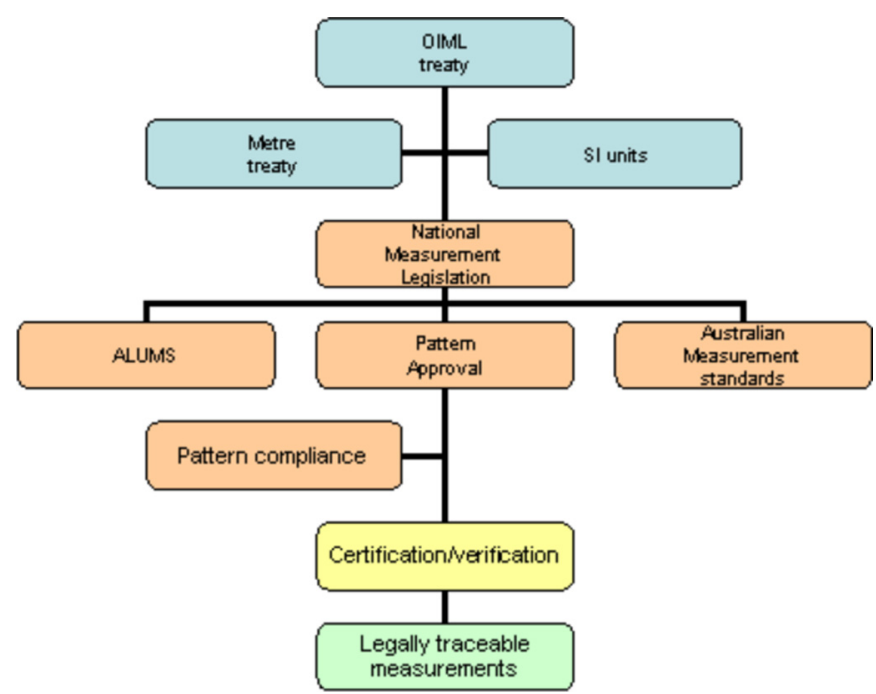

Fig. 9. Legal metrology control system (Source: OIML TC7/SC4 NIST 16-18 July 20131 Australian National Speed Measurement Program: Technology, Type Approval, Verification Eur Ing Dr Richard Brittain).

\section{Legal metrology}

LM concerns all measurements required or permitted by law. It is not specific to any technology or measurement range, for example, it is not specific to macro, micro, meso, nano or space and biotechnologies.

LM's objective, in line with OIML, aims at global harmonization of regulations and measurements. LM's scope encompasses HSE, trade and law measurements. The LM control system (Sects. 6.1 and 7) has the necessary features and capabilities to regulate the specific needs of NT. It is not concerned with terminology or threshold limits. It concerns robust and internationally harmonized measurement protocols and regulatory standards. It forbids the regulatory gaps identified by Hodge et al. The LM programme accords legal sanctions to the nanometrology programme related to HSE and trade aspects.

\subsection{Elements of a legal metrology control system}

The measurement traceability in the national measurement system outlined in Figure 6, administered through the LM control system, forms the basis for legal acceptance of measurements. It enables evidence-based regulatory reviews and decisions.

The LM control system $[27,28]$, through its certification, verification/reverification and accreditation programmes, ensures confidence and integrity of the measurement systems and accords legal status to the measurements. A LM control system ensures that measurements are what they purport to be and will satisfy Australia's international obligation and Australian law. The elements of a LM control system include (Fig. 9):

- national PA standard (written requirements based on OIML);

- PA (granted by the NMI);

- conformity to type auditing;

- uniform test procedures;

- initial certification/verification;

- subsequent certification/verification;

- use for legal purposes, that is, to make legally traceable measurements;

- verification auditing;

- independent third party accreditation of PA, verification/ certification and reverification;

- auditing of verifying/certifying laboratories; and

- training provided by NMI to the verifying authorities to ensure that they are competent to carry out verification and reverification.

\subsection{Suitability of a legal metrology control system to address the impact of nanotechnology}

The LM control system for NT measurements can - ensure measurement confidence and traceability requirements and can address legal measurements related to HSE and trade aspects of NT; 


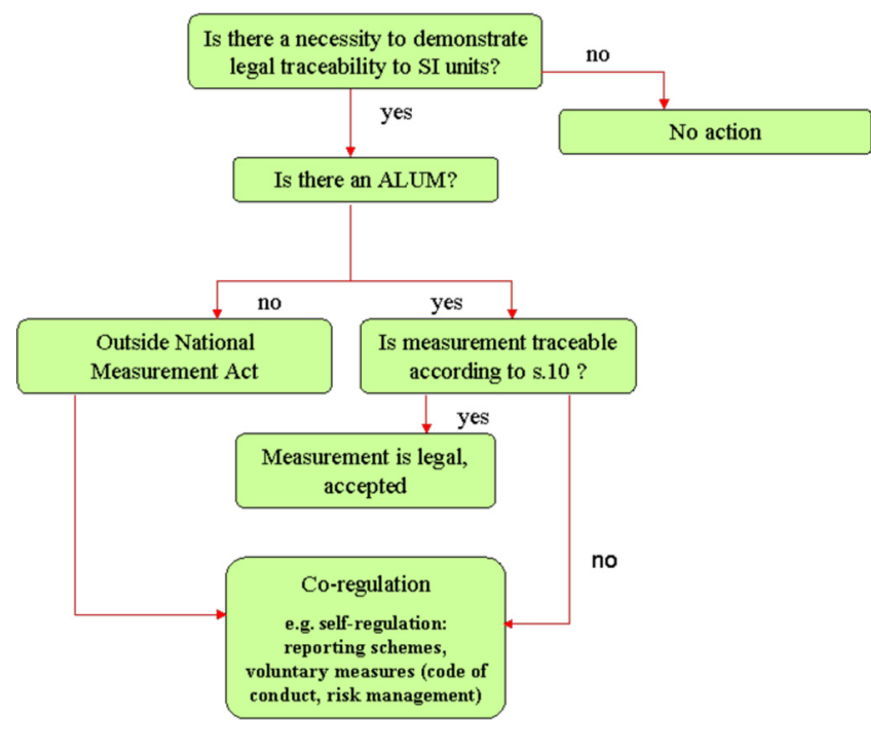

Fig. 10. Check list for traceability requirements.

prescribe Australian legal units of measurements (ALUM) for NT measurements and recognize World Health Organization (WHO)'s International Units (IU) and foreign reference standards and materials;

- provide for international harmonized standardization and recognition of NT measurements through MRA and MAA initiatives through its certification programme;

- facilitate internationally harmonized regulation for NT measurements; and

- is relevant to all legal measurement requirements: physical, chemical and biological measurement aspects of NT.

\subsection{Traceability requirement}

The Australian Government requires that development of any regulatory frameworks for NT should be founded on concrete evidence [29], hence development of a LM framework should also be based on evidence of traceability requirement as indicated in Figure 10.

At present, there is no demonstrated need for legal traceability or measurement acceptance concerning MNs; however, it is anticipated that these issues will become more important as export markets increase and clients become more astute [30].

A precautionary approach would be to ensure that the current LM control system is adequate to address specific NT measurement issues and be prepared to initiate a LMF specific to NT requirements when required. Such a LMF is not expected to be any different to the measurement requirements of its bulk counterparts in terms of prescribing maximum permissible errors (MPEs), ALUM, reference methods, reference standards and reference materials, recognizing foreign reference standards and WHO's IUs.
It should be emphasized that NT being interdisciplinary requires several measurement systems and on a case by case basis. LMFs will need to complement this requirement.

\subsection{Measurement requirements}

LMF considerations have to be coordinated through the activities of the nanometrology group, acting as the Australian coordinator of the physico-chemical HSE testing programme including eco and mammalian toxicities for $\mathrm{ZnO}$, Ceria and silver nano-particles, run by OECD's WPMN. These measurements are

- agglomeration state/aggregation;

- particle size/size distribution;

- purity and composition;

- surface area;

- surface chemistry;

- shape;

- solubility;

- porosity; and

- zeta potential.

The instruments under consideration by the nanometrology group include

- metrological SPM;

- dynamic light scattering;

- laser diffraction;

- atomic force microscope;

- optical microscopy;

- size classification (fractionation, sedimentation);

- scanning electron microscopy; and

- transmission electron microscopy.

These instruments need their parameters certified, calibrated and monitored based on the elements of the LM control system presented in Section 6.1, and subjected to PA specifications if used for trade or HSE measurements.

\subsection{Pattern approval specification}

A LMF for legal NT measurements will involve the followings:

- developing PA standards for each measurement;

- prescription of reference methods;

- prescription of reference materials;

- prescription of MPEs for PA, verification and reverification tests;

- scale interval specifications;

- reference conditions for PA, verification and reverification;

- prescription of ALUMS for chemical and biological measurements;

- recognition of units of measurement other than SI units; - prescribing conversion factors if applicable; and - prescribing sampling procedures.

\subsection{Gaps in developing LMFs}

Nanodimensional metrology forms the basis of physicochemical properties identified in Section 6.4. Demonstrating traceability by means of reference artefacts 


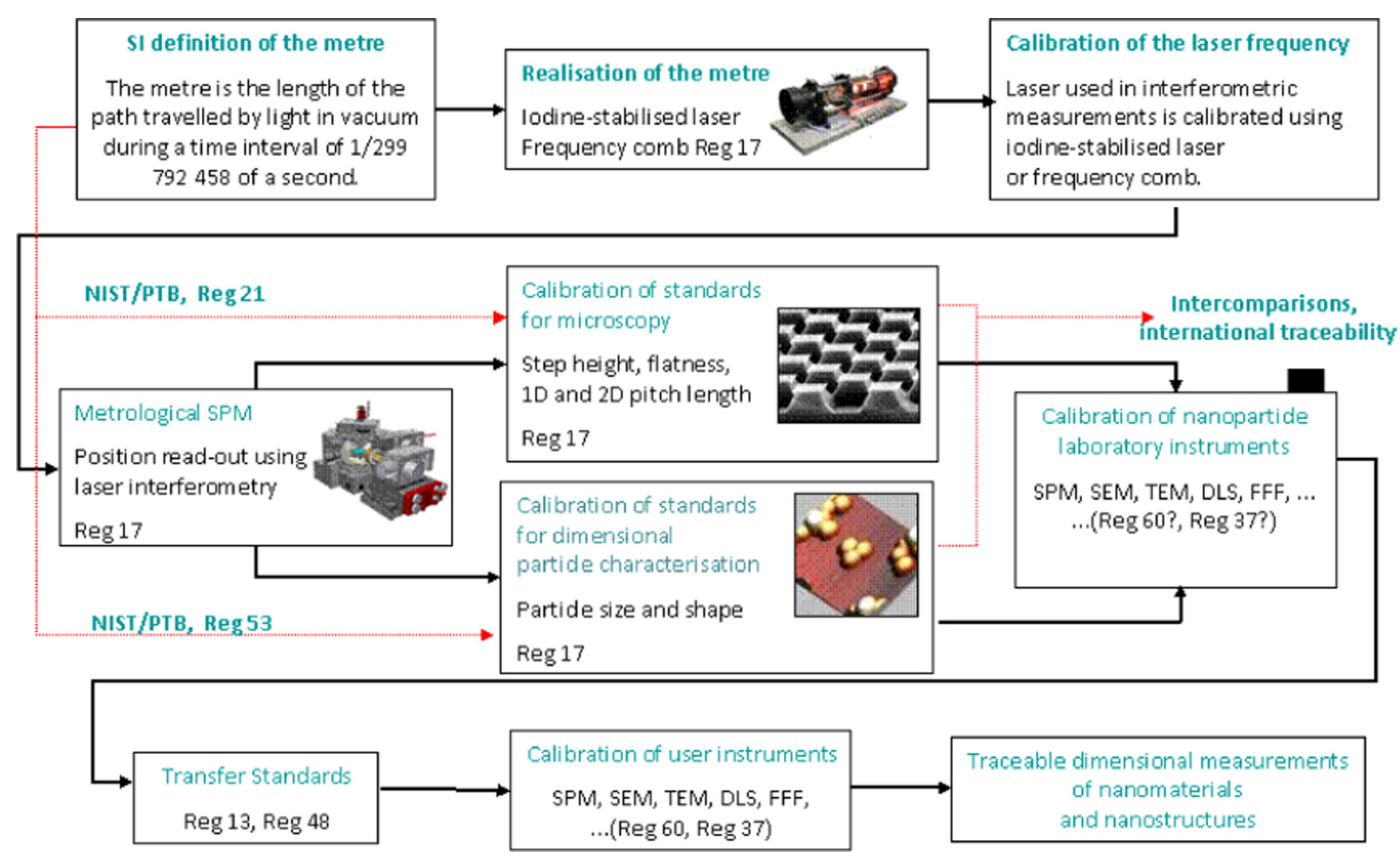

Fig. 11. A possible legal metrology framework for traceability of nano-scale dimensional measurements (Reg 17 certificates of verification of Australian primary and secondary standards of measurement; Reg 21 Recognition of foreign reference standards of measurement; Reg 37 certification of measuring instruments; Reg 48 Certification of reference materials; Reg 53 Recognition of foreign reference materials; Reg 60 Approval of patterns of measuring instruments (National Measurement Regulations $1999 \mathrm{cth})$ ).

becomes increasingly challenging as size diminishes towards the nano-scale. The development of standards for measurement, characterization and test methods for NTs and reference materials for individual measurements are still under way by the nanometrology group at the NMI. Uncertainties in measurements will need to be known to prescribe MPEs, reference methods/ standards and reference materials required for $\mathrm{PA}$ specification.

Extrinsic properties will be more challenging relative to the intrinsic properties of MNs in formulating PA specifications, as the relevant external parameters are many and unknown. It is felt that for some measurements under consideration, meeting the traceability requirements will be too complicated due to the range of external parameters that need to be considered.

In such cases, responsibility of manufacturers through co-legislation should be given serious consideration (see Sect. 3.1.4) to address the HSE issues.

During the OIML 2020 Seminar, it was proposed that in keeping with advances in technologies, the PA, certification and accreditation functions should be delegated to a private body based on a quality system (burden of proof on the manufacturers). The European Union has already adopted the scheme. Such an approach is also practised by the TGA in Australia.
The size measurement at nano-scale is inferential and is not a direct measure. Size distributions of the MNs are crucial to address the HSE issues. Size is technique specific. Study of the particle size/distribution by the author revealed that the size estimation by different instruments vary by at least $40 \%$ even in the micro-scale range [31]. Hence, appropriate conversion factors need to be worked out for different techniques to demonstrate equivalence. When the shape of the particle is not isomeric, comparisons between the techniques would be too complicated; hence, the shape factors need defining.

The following measures are recommended as immediate activities in the absence of necessary information required to develop PA:

- Prescription of ALUMS for some of the measurements identified (which would include chemical and biological measurements) and steps to recognise the WHO's IUs for biological measurements.

- Certification of Australian Primary and Secondary standards and recognition of foreign reference standards and reference materials used for nanodimensional measurements are recommended.

A possible LM framework superimposed on nanometrogy group's traceability programme for nanodimensional measurements is presented in Figure 11 ([32] is adapted for this purpose). 


\section{Conclusion}

The current LM control system is adequate to respond to the measurement needs of NT. Consideration of individual LMF for NT will be based on the following:

- on a case by case basis founded on traceability requirement;

- development should be through internationally coordinated effort;

- survey to be conducted within Australian nano-community $[33,34]$ to identify potential measurement needs requiring legal traceability and to form a LM Working Group to address the issues;

- review of the legislation to incorporate a co-legislation approach to include manufacturers'/private bodies' involvement based on a quality and risk management systems;

- inclusion of reference methods in Section 10 of the National Measurement Act (the Act);

- prescription of ALUMS for chemical measurements (if required) and recognition of WHO's IU in the Act

- LMF consideration should not be specific to $1-100 \mathrm{~nm}$, to prevent potential regulatory gaps;

- currently, HSE is being regulated by TGA, NICNAS, APVSMA and FSANZ and hence falls outside the scope LM. It is recommended that jurisdictional issues be resolved. An integrated regulatory effort, together with TGA, NICNAS and other agencies where LM will play a pivotal role in mandating legal HSE measurements, is recommended;

- Close monitoring of the developments within the eight 765 project areas of OECD WPMN (Sect. 2.4.3);

- close monitoring of the former NNS's HSE Working Group activities;

- the regulatory agencies across Australia in addition to reviewing their policies and legislations are constantly monitoring developments in NT. They are improving their technical and research capabilities, through close integration with other similar organizations across the world. It is recommended that LM adopt similar approach to be able to respond to the impact of NT;

- the extrinsic as well as the intrinsic properties of MNs need to be understood prior to develop any LMF; hence, research capabilities in this area need to be improved in close coordination with the nanometrology group.

With the increased globalization of NT, a LMF can ensure an integrated regulatory and trade approach through MRA, MAA and MOU initiatives. A LMF framework can be applied equally throughout the product lifecycle of MNs. A case-specific LMF can avert any imminent market failure, reduce transactions costs and resolve disputes based on disagreeing measurement results.

It is acknowledged that different test procedures and instruments will yield different results. The standard measurement protocols are still under development including the conversion factors to compare the results between the methods and instruments for each case. Inadequate scientific knowledge and lack of evidence for the traceability requirement prohibit any action other than to monitor the development in the area and to be prepared to respond if needed.

A consideration of individual LMF for NT should be no different from its macro or micro equivalent. Hence, the same risks and benefits apply.

However, at present, scientific knowledge related to the end application of these MNs are still being investigated and is inadequate to take any regulatory measures.

It is concluded that the LM control system is well suited to respond to the measurement requirements of NTs. A LMF for NT need will not be any different to the frameworks developed for the conventional bulk products, and hence, the same risks and benefits will apply. The study conducted so far revealed no demonstrated evidence for legal traceability requirements for any of the NT measurement. There is not sufficient information to initiate a LMF for NT at present. Hence, continued monitoring of development in NT is recommended to be able to respond if there is any need.

Discussions and contributions from Dr. R. Brittain and Dr. J. Herrmann, Dr. Dimitrios Georgakopoulos, NMI Australia are gratefully acknowledged.

\section{References}

1. http://www.rncos.com/Market-Analysis-Reports/Nano technology-Market-Outlook-2020-IM687.htm

2. L.D. Williams, W. Adams, Nanotechnology demystified (McGraw-Hill, 2006), Vol. 174

3. http://esc.u-strasbg.fr/docs/2012/lectures/Braun_A.pptx

4. http://www.nanotechproject.org/inventories/consumer/ analysis draft

5. G. Hodge, D. Bowman, K. Ludlow, New global frontiers in regulation (Edward Elgar Publishing, Inc, 2007)

6. E. Mantovani, A. Porcari, Developments in nanotechnologies regulation and standards, NMP - nanosciences, nanotechnologies, materials and new production technologies (2009), http://www.observatorynano.eu/

7. J.J. Ramsden, What is nanotechnology, Nanotechnol. Percept. 1, 3 (2005)

8. http://www.ringsurf.com/online/2009-top_down.html

9. http://www.lanl.gov/mst/nano/definition.h̆tml

10. NSERC Nano Innovation Platform, http://www.physics. mcgill.ca/NSERCnanoIP/e/awards/awards_2005.html

11. Living in a post-chemistry world - the regulatory challenges of emerging nanotechnologies (2020 Science, 2009), http://2020science.org/2009/09/11/living-in-a-post-chemis try-world-the-regulatory-challenges-of-emerging-nanotech nologies/

12. D.L. Beke, S. Szabo, M. Kis-Varga, Intrinsic and domain magnetism in nanomaterials, Adv. Condens. Matter Mater. Res. 5, 77 (2004)

13. ISO/TS 27687, Draft 2007

14. G. Oberdörster, A. Maynard, K. Donaldson, V. Castranova, J. Fitzpatrick, K. Ausman, J. Carter, B. Karn, W. Kreyling, D. Lai, S. Olin, N. Monteiro-Riviere, D. Warheit, H. Yang, Principles for characterizing the potential human health effects from exposure to nanomaterials: Elements of a 
screening strategy, Part. Fibre Toxicol. 2, 8 (2005), http://www.particleandfibretoxicology.com/content/2/1/ 8, downloaded 08-01-01

15. M. Auffan, J. Rose, J.-Y. Bottero, G.V. Lowry, J.-P. Jolivet, M. R. Wiesner, Towards a definition of inorganic nanoparticles from an environmental, health and safety perspective, Nat. Nanotechnol. 4 (2009), www.nature.com/naturenanotechnology

16. L.-J. Schierow, Engineered nano-scale materials and derivative products: regulatory challenges, CRS report for congress (January 22, 2008), http://www.fas.org/sgp/crs/misc/RL34332.pdf

17. J.C. Davies, Oversight of next generation nanotechnology (PEN 18, 2009)

18. http://en.wikipedia.org/wiki/Regulation_of_nanotechnolo gy\#cite_note-Futures-12\#cite_note-Futures-12

19. G. Merchant, D. Sylvester, K.W. Abbott, in Nanotechnology regulation: the United States Approach, new global frontiers in regulation, edited by G. Hodge, D. Bowman, K. Ludlow (Edgar Elgar, USA, 2007)

20. http://admin.umt.edu.pk/Media/Site/STD/FileManager/ OsamaArticle/August2015/10august/godadmin-A-10-2-13d7f887d.pdf

21. K. Donaldson, V. Stone, C.L. Tran, W. Kreyling, P.J.A Born, Nanotoxicology, Occup. Environ. Med. 61, 727 (2004)

22. D.J. Whitehouse, Nanometrology, Contemp. Phys. 49, 351 (2008)

23. http://admin.umt.edu.pk/Media/Site/STD/FileManager/ OsamaArticle/August2015/10august/godadmin-A-10-2-13d7f887d.pdf

24. J. Miles, The metrology of nanoparticles, Therapeutic Goods Administration, Nanotechnology training program, Canberra, 04-06 May 2009 (2009)

25. J. Miles, Written statement for inquiry into Nanotechnology in NSW (May 2008)

26. G.N. Peggs, Measurement in the nanoworld, Nanotechnol. Percept. 1, 18 (2005)

27. J. Birch, Metrological control of measuring instruments, Leaflet 26 (1998)

28. R. Brittain, Presentation: Introduction to legal metrology (February 2009)
29. http://www.innovation.gov.au/Industry/Nanotechnology/ Documents/ObjectivesPaper.pdf

30. J. Miles, Nanometrology: The critical role of measurement in supporting Australian nanotechnology (NMI TR 12, 2006), first edition

31. S. Devasahayam, Particle sizing and fractionation: The methods and benefits of fine grinding ores- final report, AMIRA project P336A (JKMRC, University of Queensland, Brisbane, Australia, 1997), p. 125

32. http://www.safeworkaustralia.gov.au/sites/SWA/whs-in formation/Nanotechnology/Documents/Nanometrology DocumentaryStandardsNanotechnology.pdf

33. http://www.nanowerk.com/nanotechnology/Nanotechnology_ Companies in Australia.php

34. http://www.ausnano.net/

35. M.E. Vance, T. Kuiken, E.P. Vejerano, S.P. McGinnis, M.F. Hochella Jr., D. Rejeski, M.S. Hull, Nanotechnology in the real world: Redeveloping the nanomaterial consumer products inventory, Beilstein J. Nanotechnol. 6, 1769 (2015), doi:10.3762/bjnano.6.181. http://www.beilstein-journals. org/bjnano/content/pdf/2190-4286-6-181.pdf

36. R.W. Whatmore, Nanotechnology: big prospects for small engineering, Ingeria 9, 28-34 (2001), www.nanotec.org.uk/ report/chapter4.pdf

37. R.W. Whatmore, Nanotechnology-should we be worried? Nanotechnol. Percept. I, 67 (2005)

38. S.G. Royce, D. Mukherjee, T. Cai, S.S. Xu, J.A. Alexander, Z. Mi, L. Calderon, G. Mainelis, K. Lee, P.J. Lioy, T.D. Tetley, K.F. Chung, J. Zhang, P.G. Georgopoulos, Modeling population exposures to silver nanoparticles present in consumer products. J. Nanopart. Res. 16, 2724 (2014), doi:10.1007/s11051-014-2724-4. PMCID: PMC4346165. http:// ccl.rutgers.edu/ccl-researchers-present-at-nanoparticlestudies-at-duke-university/

39. J.J. Ramsden, J. Freeman, The nano-scale, Nanotechnol. Percept. 5 (2009)

40. https://www.researchgate.net/file.PostFileLoader.html? $\mathrm{id}=573232405 \mathrm{~b} 49524 \mathrm{ec} 80 \mathrm{~d} 7413 \&$ assetKey $=\mathrm{AS} \%$ 3A360274138812416\%401462907456896

Cite this article as: Sheila Devasahayam, Overview of an internationally integrated nanotechnology governance, Int. J. Metrol. Qual. Eng. 8, 8 (2017) 